\title{
ANÁLISE DA VIABILIDADE ECONÔMICO-FINANCEIRA DE UMA INDÚSTRIA DESTINADA AO BENEFICIAMENTO DE RECICLÁVEIS
}

\author{
Hévilla Souza Oliveira (Universidade Federal do Ceará) hevillasouza@alu.ufc.br \\ Daiane de Oliveira Costa (Universidade Federal do Ceará) daiane_oliveirac@ hotmail.com \\ Rayane Araújo Lima (Universidade Federal do Ceará) rayanearaujo@alu.ufc.br \\ Sâmara Margarida Santana Soares (Universidade Federal do Ceará) \\ smargarida-@hotmail.com \\ Carla Amanda Matos Lima (Universidade Federal do Ceará) amandamts1@alu.ufc.br
}

\section{Resumo}

Atualmente, devido à forte propagação de informações sobre os impactos causados à natureza, os indivíduos apresentam-se bem mais preocupados com o meio em que vivem, gerando uma tendência mundial de melhores práticas, como a reciclagem de mateirias. Um dos setores onde se evidencia grande utilização de plásticos é o da agricultura, dados os benefícios destes, como a preservação do solo, auxilio no controle de temperatura, redução da incidência de parasitas, etc. Entretanto, mesmo com suas vantagens, o aumento da sua utilização nesse ramo e a necessidade de um descarte correto desse material, configura-se como um problema ambiental. Assim, uma das melhores hipóteses para a solução dessa questão é a reciclagem. O presente trabalho, portanto, objetiva realizar um estudo de viabilidade econômico-financeira para a análise do retorno do investimento da aquisição de equipamentos, bem como a infraestrutura necessária para o beneficiamento de TNT e Mulching aplicados à agricultura. Por meio do estudo de conceitos e indicadores econômicos e financeiros, foi estudada a viabilidade para o projeto em questão. A relevância deste estudo está não apenas na propagação e aquisição do conhecimento no que diz respeito a aspectos financeiros, como também retrata a importância da elaboração de processos industriais capazes de proporcionar a reutilização de materiais suscetíveis a reciclagem.

Palavras-Chaves: Análise Financeira de Investimento. VPL. TIR. Pay-back descontado. Beneficiamento de recicláveis.

\section{Introdução}

Atualmente, devido à forte propagação de informações sobre os impactos causados à natureza pelo consumismo, os indivíduos apresentam-se bem mais preocupados com o meio em que 
vivem, gerando uma tendência mundial de melhores práticas, como a reciclagem de produtos. Entende-se por reciclagem o ato de separar materiais do lixo, dentre eles, papéis, plásticos, vidros e metais, os quais através de ações humanas são transformados e introduzidos novamente no ciclo do mercado de consumo (CINQUETTI, 2004).

Um dos setores onde se evidencia grande utilização de plásticos é o da agricultura. De acordo com Goto (1997), a utilização do plástico neste setor é antiga, iniciando-se na década de 70, quando foi empregada pela primeira vez em grande escala no Brasil com o uso do mulching na cultura do morango. Santos (2014) afirma que a utilização de plásticos propicia diversas vantagens, pois ajuda na manutenção da humidade do solo, facilita a aplicação de fertilizantes, repele certos insetos, mantém uma maior temperatura no solo durante a noite, dentre outras.

Embora seja benéfico do ponto de vista agronômico, o descarte desses materiais após o uso configura-se como um problema ambiental, ainda mais considerando-se o crescente aumento da sua utilização, que chega a cerca de 25 mil hectares de terra desse tipo de agricultura no Brasil (EMBRAPA, 2015). Dessa forma, a reciclagem apresenta-se como uma boa alternativa para a resolução deste problema. De acordo com o Instituto de Pesquisa Econômica Aplicada (IPEA) (2017), este é um setor ainda pouco explorado no Brasil, uma vez que apenas $13 \%$ de resíduos sólidos tem como destino a reciclagem.

Diante do exposto, observa-se uma oportunidade de negócio a ser estudada. No entanto, quando se pretende iniciar um novo empreendimento é de fundamental importância analisar a viabilidade do projeto de investimento, que inclui diversos indicadores econômicos e financeiros. Neste sentido, o presente trabalho tem como objetivo realizar uma análise da viabilidade econômico-financeira para uma empresa destinada ao beneficiamento de plásticos aplicados na agricultura, com localização na cidade de Mossoró (RN).

\section{Caracterização do empreendimento}

A cidade de Mossoró, onde ficará localizada a empresa, está no centro de uma região onde o agronegócio encontra-se em expansão (SILVA, 2015). Oliveira et al. (2013) aponta a cidade como a principal produtora de melão do estado do Rio Grande do Norte, onde ficam localizadas algumas empresas líderes neste mercado, as quais serão tomadas como fornecedoras de matériaprima para o beneficiamento do plástico usado no cultivo, principal atividade a ser desenvolvida. 
A técnica de beneficiamento dos materiais recicláveis promove a estes, condições que possibilitem o seu uso, seja como matéria-prima ou produto, agregando valor para avança-lo nos elos da cadeia produtiva. Inicialmente, a empresa se restringirá a dois tipos de plásticos, o TNT e o Mulching, os quais são bastante presentes no cultivo das empresas localizadas próximo à região onde a empresa será instalada. O TNT é utilizado em cobertura de canteiros de fumo, na fase de formação de mudas. Já o mulching é um filme plástico de baixo custo, que protege o solo e o sistema radicular das plantas, utilizado para fazer o revestimento da área de plantio.

O processo de beneficiamento deve ocorrer de forma que garanta a qualidade do material, utilizando-se, para isso, equipamentos adequados para realizar procedimentos como triagem, moagem e prensagem, em busca de uma qualidade que atenda aos padrões do mercado e proporcione a comercialização diretamente com indústrias de transformação de plásticos, localizadas em todo país.

A organização contará com um quadro de 25 funcionários sendo assim distribuídos: 21 auxiliares de produção, 1 empilhador, 2 supervisores de produção, e 1 motorista de caminhão responsável pela retirada de matéria-prima nas fazendas. A proprietária ficará responsável por toda parte administrativa da empresa.

Como pontos fortes para o estabelecimento da empresa nesta região, destaca-se: a qualidade do produto, boa localização em relação aos fornecedores, boas instalações e estrutura física, preço de venda competitivo, baixo custo para aquisição de matéria-prima, produto sustentável, forte apelo ecológico e poucos concorrentes na região disputando a matéria-prima.

\section{Referencial teórico}

\subsection{Investimento}

Para que uma empresa consiga realizar alterações em sua estrutura física e tecnológica, bem como elevar a qualidade de sua mão de obra, ela deve realizar investimentos. O investimento é todo gasto realizado baseado na projeção de benefícios futuros, levando-se em conta a função de sua vida útil (PADOVEZE, 2006; MARTINS, 2003). Portanto pode-se determinar este termo como uma aplicação monetária realizada no intuito de alcançar proventos financeiros futuros. 


\subsection{Receita}

A receita é um montante obtido através da venda de bens ou serviços, definida através da Equação 1 (MARTINS, 2003).

$$
\begin{gathered}
\text { Receita }=\text { quantidade de produtos vendidos } \times \\
\text { preço unitário do produtos }
\end{gathered}
$$

\subsection{Custos e despesas}

Custos e despesas são dois gastos presentes em todas as organizações. Eles diferenciam-se entre si devido ao seu grau de relação com o produto. Enquanto os custos estão diretamente ligados à produção do item, as despesas estão associadas aos gastos obtidos com a venda e distribuição deste (PADOVEZE, 2006).

Ambos são subdivididos em duas classificações: fixos e variáveis. Os custos e despesas variáveis são aqueles que podem variar de acordo com o volume produzido, enquanto os custos e despesas fixas permanecem inalterados diante deste mesmo aspecto (MARTINS, 2003).

\subsection{Ponto de Equilíbrio}

Em relação aos parâmetros que auxiliam os gestores na identificação da saúde financeira das organizações, temos os pontos de equilíbrio. De acordo com Marion e Ribeiro (2011), o Ponto de Equilíbrio Contábil (PEC) (Equação 2), mostra o patamar em que a margem de contribuição dos produtos (Equação 3) coincide com os custos e despesas fixas da empresa.

$$
P E_{U . M . C o n t a ́ b i l}=\frac{\mathrm{CFT}+\mathrm{DFT}}{\mathrm{MCT} \%}
$$

Onde:

$$
M C T \%=\frac{\sum M C U_{i}}{\sum P V_{i}}
$$

Outro indicador comumente empregado é o Ponto de Equilíbrio Econômico (PEE), expresso de acordo com Martins (2003) pela Equação 4.

$$
P E_{U . M . E c o n o ̂ m i c o}=\frac{\mathrm{CFT}+\mathrm{DFT}+\text { Lucro mínimo desejado }}{\mathrm{MCT} \%}
$$


Em relação ao Ponto de Equilíbrio Financeiro (PEF), este se difere dos demais indicadores pela necessidade da subtração da depreciação de máquinas e equipamentos em relação aos custos e despesas fixas, sendo definido pela Equação 5 (MARTINS, 2003).

$$
P E_{U . M . F i n a n c e i r o}=\frac{\mathrm{CFT}+\mathrm{DFT}-\text { Depreciação }}{\mathrm{MCT} \%}
$$

\subsection{Demonstrativo do Resultado do Exercício}

Com o intuito de analisar a situação financeira das organizações, a Demonstração do Resultado do Exercício é empregada em um ciclo contábil geralmente de um ano para a visualização de um possível lucro ou prejuízo. Essa demonstração financeira é composta pelo o estudo da receita, gastos fixos e variáveis e retenção de impostos a fim de se obter o resultado líquido do exercício (ASSAF NETO; LIMA, 2014).

\subsection{Fluxo de Caixa Livre}

O Fluxo de Caixa Livre (FCL) auxilia na visualização da rentabilidade de investimentos, possíveis benefícios e a situação financeira da instituição decorrente das suas entradas e saídas. De acordo com Samanez (2012), a construção do FCL é composta por aspectos relacionados ao investimento (aquisição de ativos, necessidade de capital de giro, etc.), em relação ao fluxo operacional (receitas, custos, despesas, impostos, dentre outros) e pode englobar também elementos de financiamento (a exemplo o valor da amortização, juros e os benefícios fiscais).

\subsection{Lucratividade e rentabilidade}

Lucratividade e rentabilidade são índices que indicam o nível de sucesso de um investimento. Enquanto lucratividade é o conceito de lucro sobre vendas (Equação 6), rentabilidade pode ser definida como o rendimento do capital investido, como aponta a Equação 7 (PADOVEZE, 2006).

$$
\begin{gathered}
\text { Lucratividade }=\frac{\text { Lucro líquido }}{\text { Faturamento Bruto }} \\
\text { Rentabilidade }=\frac{\text { Lucro líquido }}{\text { Investimento }}
\end{gathered}
$$

\subsection{VPL, TIR e pay-back descontado}

VPL, TIR e pay-back descontado são indicadores utilizados para a análise da viabilidade econômica de um investimento e que foram aplicados no presente estudo. 
O Valor Presente Líquido (VPL) possui o objetivo de avaliar em termos de valor presentes os impactos futuros associados a um investimento ou projeto. Sua principal finalidade é apontar quais investimento são mais valiosos do ponto de vista de seus patrocinadores. $\mathrm{O}$ critério de decisão consiste em: se o VPL > 0, o projeto é economicamente viável e pode ser calculado de acordo com a Equação 8 (SAMANEZ, 2002).

$$
V P L=-\mathrm{I}+\sum_{t=1}^{n} \frac{F C_{t}}{(1+K)^{t}}
$$

Onde,

$\mathrm{I}=$ investimento inicial

$\mathrm{FC}_{\mathrm{t}}=$ fluxo de caixa livre o t-ésimo período

$\mathrm{K}=$ custo do capital

A Taxa Interna de Retorno (TIR) é a taxa de retorno esperada para determinado investimento. Diferentemente do VPL, a TIR não possui a finalidade de avaliar rentabilidade, seu objetivo é apenas apontar a taxa intrínseca de rendimento. Por ser uma taxa hipotética de desconto que anula o VPL, o critério de decisão será: se $^{*}>\mathrm{K}$, o projeto é economicamente viável. A Equação 9 define o cálculo da TIR (valor de i* que satisfaz a equação) (SAMANEZ, 2002).

$$
V P L=-\mathrm{I}+\sum_{t=1}^{n} \frac{F C_{t}}{\left(1+i^{*}\right)}=0
$$

Onde,

$\mathrm{I}=$ investimento inicial

$\mathrm{FC}_{\mathrm{t}}=$ fluxo de caixa livre o t-ésimo período

$i^{*}=\mathrm{TIR}=$ taxa interna de retorno quando $\mathrm{VPL}=0$

Já o pay-back descontado é o indicador utilizado para definir o período de tempo necessário para recuperar o capital investido (o valor de T na Equação 10). Sua maior utilidade é na avaliação de diversas opções de investimento, geralmente é um indicador complementar ao VPL (SAMANEZ, 2002). 


$$
I=\sum_{t=1}^{T} \frac{F C_{t}}{(1+K)^{t}}
$$

Onde,

$\mathrm{I}=$ investimento inicial

$\mathrm{FC}_{\mathrm{t}}=$ fluxo de caixa livre o t-ésimo período

$\mathrm{K}$ = custo de capital

\section{Metodologia}

\subsection{Caracterização da pesquisa}

O presente trabalho possui como procedimento metodológico a pesquisa bibliográfica, que permitiu embasar o conhecimento dos autores quanto ao conteúdo; a coletada de dados, dada a análise documental de arquivos pertencentes à organização; e o estudo de caso, visto que a pesquisa corroborou com a compreensão de fatores individuais pertencentes à organização (YIN, 2001). A natureza é aplicada, pois objetiva proporcionar conhecimentos de aplicação prática voltados à solução do problema em específico (GERHARDT e SILVEIRA, 2009),

A abordagem do estudo se classificou como quantitativa dado que as ferramentas utilizadas foram baseadas em números, e as informações coletadas foram classificadas e analisadas. Adiante construiu-se hipóteses e opiniões para a interpretação e relação das variáveis, por meio do qual foi possível alcançar os resultados pretendidos (PRODANOV e FREITAS, 2013). Quanto aos objetivos, a pesquisa se caracteriza como exploratória pois é realizada com o propósito de modificar e explicar conceitos, visando a elaboração de problemas e hipóteses (GIL, 2008).

\subsection{Coleta de dados}

A coleta dos dados iniciou-se com uma pesquisa sobre o mercado, onde foi notada a oportunidade para investir em um novo ramo, sendo este o beneficiamento dos plásticos TNT e Mulching. Determinou-se esse nicho devido à qualidade garantida de que as sucatas se encontram próprias para a reciclagem e o preço destas ser acessível, além da matéria-prima ser proveniente de fazendas próximas ao local onde a empresa será instalada. 
Dessa forma, foram realizadas pesquisas de mercado sobre o preço da comercialização desses produtos, a demanda média que seria absorvida pelo mercado e os principais fornecedores, bem como a listagem dos equipamentos e o investimento necessário para o alcance desse propósito. Posteriormente, realizou-se o cálculo da receita com base na demanda média, o levantamento do enquadramento tributário e os custos/despesas fixas e variáveis. Com base nestes, tornou-se possível a determinação de indicadores como os pontos de equilíbrio e as investigações da saúde da organização por meio da DRE e o fluxo de caixa livre. Para os cálculos foram considerados dois cenários, devido à diferença presente no período de depreciação dos equipamentos: o primeiro em relação aos 5 primeiros anos, nos quais constam a depreciação do caminhão; e o segundo, referente aos 5 anos seguintes, desconsiderando este valor.

Indicadores como o VPL, TIR e pay-back descontado foram utilizados para avaliar a viabilidade do empreendimento. Ademais, analisou-se a rentabilidade e o retorno esperado, encerrando o estudo com as considerações finais e as proposições de estudos futuros.

\section{Análise econômico-financeira}

De acordo com o faturamento bruto esperado, o enquadramento tributário da empresa é o regime Simples Nacional, que apresenta, segundo a sua tabela de impostos, a alíquota incidente sobre os preços de venda de $11,2 \%$, porcentagem referente ao ano de 2020 para a categoria industrial.

\subsection{Investimento}

O investimento aqui pretendido é o beneficiamento de dois tipos de materiais oriundos de empresas agrícolas, são estes o TNT e o Mulching. Para isso é necessário infraestrutura, maquinário e mão de obra, portanto os gastos advindos do investimento são os apresentados nos Quadros 1 ao 4. 
Quadro 1 - Investimentos fixos

\begin{tabular}{|c|c|c|c|c|c|}
\hline \multicolumn{6}{|c|}{ Investimentos Fixos } \\
\hline \multirow[t]{2}{*}{ Descrição } & \multicolumn{2}{|c|}{ Valor Unitário } & \multirow{2}{*}{$\begin{array}{c}\text { Quant. } \\
10\end{array}$} & \multicolumn{2}{|c|}{ Valor Total } \\
\hline & $\mathrm{R} \$$ & 40,00 & & $\mathrm{R} \$$ & 400,00 \\
\hline Máquina de corte & $\mathrm{R} \$$ & 700,00 & 6 & $\mathrm{R} \$$ & $4.200,00$ \\
\hline Balança Industrial & $\mathrm{R} \$$ & $1.700,00$ & 1 & $\mathrm{R} \$$ & $1.700,00$ \\
\hline Esteira Industrial para transporte & $\mathrm{R} \$$ & $7.800,00$ & 1 & $\mathrm{R} \$$ & $7.800,00$ \\
\hline Kit (Moinho + Ventoinhas + Centrifugador) & $\mathrm{R} \$$ & $250.000,00$ & 1 & $\mathrm{R} \$$ & $250.000,00$ \\
\hline Amolador de Lâminas para Moinho & $\mathrm{R} \$$ & $2.500,00$ & 1 & $\mathrm{R} \$$ & $2.500,00$ \\
\hline Prensa Enfardadeira & $\mathrm{R} \$$ & $45.000,00$ & 2 & $\mathrm{R} \$$ & $90.000,00$ \\
\hline Cadeira para escritório & $\mathrm{R} \$$ & 367,00 & 1 & $\mathrm{R} \$$ & 367,00 \\
\hline Mesa para escritório & $\mathrm{R} \$$ & 399,00 & 1 & $\mathrm{R} \$$ & 399,00 \\
\hline Armário & $\mathrm{R} \$$ & 319,00 & 1 & $\mathrm{R} \$$ & 319,00 \\
\hline Ar-condicionado & $\mathrm{R} \$$ & $1.166,00$ & 1 & $\mathrm{R} \$$ & $1.166,00$ \\
\hline Telefone & $\mathrm{R} \$$ & 109,00 & 1 & $\mathrm{R} \$$ & 109,00 \\
\hline Computador & $\mathrm{R} \$$ & $1.699,00$ & 1 & $\mathrm{R} \$$ & $1.699,00$ \\
\hline Caminhão (Incluso caçambas e garra) & $\mathrm{R} \$$ & $474.000,00$ & 1 & $\mathrm{R} \$$ & $474.000,00$ \\
\hline Total & & - & - & $\mathbf{R S S}$ & $834.659,00$ \\
\hline
\end{tabular}

Fonte: Autores (2020)

Quadro 2 - Investimentos pré-operacionais

\begin{tabular}{|l|lr|}
\hline \multicolumn{2}{|c|}{ Investimentos Pré-Operacionais } \\
\hline \multicolumn{1}{|c|}{ Descrição } & \multicolumn{2}{c|}{ Valor } \\
\hline Execução do Projeto de Licença Ambiental & $\mathrm{R} \$$ & $7.000,00$ \\
\hline Execução do Projeto de Instalaç̃̃es Elétricas & $\mathrm{R} \$$ & $45.300,00$ \\
\hline Execução do Projeto do Corpo de Bombeiros & $\mathrm{R} \$$ & $9.000,00$ \\
\hline Total & $\mathrm{R} \$$ & $\mathbf{6 1 . 3 0 0 , 0 0}$ \\
\hline
\end{tabular}

Fonte: Autores (2020) 
Quadro 3 - Capital de giro

\begin{tabular}{|l|lr|}
\hline \multicolumn{3}{|c|}{ Contas de Capital de Giro } \\
\hline Descrição & \multicolumn{2}{c|}{ Valor } \\
\hline Estoque Inicial de TNT & $\mathrm{R} \$$ & $6.000,00$ \\
\hline Estoque Inicial de Mulching & $\mathrm{R} \$$ & $10.200,00$ \\
\hline Salário do Motorista do Caminhão & $\mathrm{R} \$$ & $4.000,00$ \\
\hline Salário dos Supervisores de Produção & $\mathrm{R} \$$ & $3.000,00$ \\
\hline Salário dos Auxiliares de Produção & $\mathrm{R} \$$ & $21.945,00$ \\
\hline Salário do Empilhador & $\mathrm{R} \$$ & $1.045,00$ \\
\hline Água & $\mathrm{R} \$$ & 430,00 \\
\hline Telefone & $\mathrm{R} \$$ & 455,00 \\
\hline Internet & $\mathrm{R} \$$ & 50,00 \\
\hline Material de Limpeza & $\mathrm{R} \$$ & 65,00 \\
\hline Material de Escritório & $\mathrm{R} \$$ & 122,00 \\
\hline Pesagem de Cargas (Terceirizado) & $\mathrm{R} \$$ & 80,00 \\
\hline Aluguel do Prédio Administrativo & $\mathrm{R} \$$ & 500,00 \\
\hline Energia Elétrica do Prédio Administrativo & $\mathrm{R} \$$ & 524,00 \\
\hline Total & $\mathrm{R} \$$ & $\mathbf{4 8 . 4 1 6 , 0 0}$ \\
\hline
\end{tabular}

Fonte: Autores (2020)

Quadro 4 - Investimento total

\begin{tabular}{|l|lc|}
\hline \multicolumn{2}{|c|}{ Investimento Total } \\
\hline \multicolumn{2}{|c|}{ Descrição } & \multicolumn{2}{c|}{ Valor } \\
\hline Investimentos Fixos & R\$ & $834.659,00$ \\
\hline Investimentos Pré-Operacionais & R\$ & $61.300,00$ \\
\hline Necessidade de Capital de Giro & R\$ & $48.416,00$ \\
\hline Total & R\$ & $\mathbf{9 4 4 . 3 7 5 , 0 0}$ \\
\hline
\end{tabular}

Fonte: Autores (2020)

\subsection{Receita}

A receita (Quadro 5) será obtida por meio da venda do quilo dos produtos, onde estimou-se a demanda por meio de uma análise de mercado.

Quadro 5 - Receitas mensais e anuais

\begin{tabular}{|c|c|c|c|c|c|}
\hline \multicolumn{6}{|c|}{ Receitas } \\
\hline Produto & Qt Estimada de Venda ( $\mathrm{kg}$ ) & & $\mathrm{Kg}$ & & ceita Bruta \\
\hline TNT & 24.000 & $\mathrm{RSS}$ & 1,70 & $\mathrm{RSS}$ & $40.800,00$ \\
\hline Mulching & 60.000 & RSS & 1,80 & $\mathrm{RSS}$ & $108.000,00$ \\
\hline \multicolumn{4}{|c|}{ Total Mensal } & $\mathrm{R} \$$ & $148.800,00$ \\
\hline \multicolumn{4}{|c|}{ Total Anual } & $\mathrm{RS}$ & $1.785 .600,00$ \\
\hline
\end{tabular}

Fonte: Autores (2020)

\subsection{Custos e despesas}

A depreciação, os custos e as despesas (fixos e variáveis) são apresentados nos Quadros 6 a 10. 
Quadro 6 - Depreciação

\begin{tabular}{|c|c|c|c|c|c|c|c|c|}
\hline \multicolumn{9}{|c|}{ Depreciação } \\
\hline Equipamentos & \multicolumn{2}{|c|}{ Valor Unitário } & Quantidade & Vida Útil (Ano) & \multicolumn{2}{|c|}{$\begin{array}{l}\text { Depreciação (Anual) } \\
\text { CENÁRIO } 1\end{array}$} & \multicolumn{2}{|c|}{$\begin{array}{c}\text { Depreciação (Anual) } \\
\text { CENÁRIO } 2\end{array}$} \\
\hline Máquina de corte & $\mathrm{RS}$ & 700,00 & 6 & 10 & $\mathrm{R} \$$ & 420,00 & $\mathrm{R} \$$ & 420,00 \\
\hline Balança industrial & $\mathrm{R} \$$ & $1.700,00$ & 1 & 10 & $\mathrm{R} \$$ & 170,00 & $\mathrm{RS}$ & 170,00 \\
\hline Esteira & $\mathrm{RS}$ & $7.800,00$ & 1 & 10 & $\mathrm{RS}$ & 780,00 & $\mathrm{RS}$ & 780,00 \\
\hline Kit Moinho + Ventoinhas + Centrifugador & $\mathrm{RS}$ & $250.000,00$ & 1 & 10 & $\mathrm{RS}$ & $25.000,00$ & $\mathrm{RS}$ & $25.000,00$ \\
\hline Amolador de Lâminas para Moinho & $\mathrm{RS}$ & $2.500,00$ & 1 & 10 & $\mathrm{RS}$ & 250,00 & $\mathrm{RS}$ & 250,00 \\
\hline Prensa Enfardadora & $\mathrm{RS}$ & $45.000,00$ & 2 & 10 & $\mathrm{RS}$ & $9.000,00$ & $\mathrm{RS}$ & $9.000,00$ \\
\hline Caminhão & $\mathrm{R} \$$ & $474.000,00$ & 1 & 5 & $\mathrm{R} \$$ & $94.800,00$ & $\mathrm{RS}$ & - \\
\hline \multicolumn{5}{|c|}{ Total } & $\mathrm{R} \$$ & $130.420,00$ & $\mathrm{R} \$$ & $35.620,00$ \\
\hline
\end{tabular}

Fonte: Autores (2020)

Como pode-se observar o valor da depreciação é variável (caminhão 5 anos e os demais equipamentos 10 anos). Portanto, realizou-se os cálculos para dois cenários, onde o cenário 1 refere-se aos primeiros cinco anos e contempla a depreciação de todos os equipamentos incluindo o caminhão. Já o cenário 2 consiste na análise dos cinco anos seguintes, referentes ao resto do período da depreciação dos demais equipamentos.

Quadro 7 - Custos fixos mensais e anuais

\begin{tabular}{|c|c|c|c|c|c|c|}
\hline \multicolumn{7}{|c|}{ Custos Fixos } \\
\hline Descrição & & ปÁRIO 1 & Valc & ICENÁRIO 1 & & ENÁRIO 2 \\
\hline Seguro do Caminhão & $\mathrm{R} \$$ & $4.021,00$ & $\mathrm{RS}$ & $48.252,00$ & $\mathrm{RS}$ & $48.252,00$ \\
\hline Combustivel e Manutenção do Caminhão & RS & $3.855,07$ & $\mathrm{RS}$ & $46.260,84$ & $\mathrm{RS}$ & $46.260,84$ \\
\hline Transporte dos Funcionários da Produção & $\mathrm{RS}$ & $2.500,00$ & $\mathrm{RS}$ & $30.000,00$ & $\mathrm{RS}$ & $30.000,00$ \\
\hline Equipamento de Proteção Individual (EPI) & $\mathrm{R} \$$ & 360,00 & $R S$ & $4.320,00$ & $\mathrm{RS}$ & $4.320,00$ \\
\hline Manutenção dos Equipamentos & $\mathrm{RS}$ & $1.000,00$ & $\mathrm{RS}$ & $12.000,00$ & $\mathrm{RS}$ & $12.000,00$ \\
\hline Energia Elétrica da Fábrica & $\mathrm{RS}$ & $3.306,03$ & $\mathrm{RS}$ & $39.672,36$ & $\mathrm{RS}$ & $39.672,36$ \\
\hline Aluguel da Empilhadeira & $\mathrm{RS}$ & $2.000,00$ & $\mathrm{RS}$ & $24.000,00$ & $\mathrm{RS}$ & $24.000,00$ \\
\hline Gás para Empilhadeira & $\mathrm{RS}$ & 325,00 & $\mathrm{RS}$ & $3.900,00$ & $\mathrm{RS}$ & $3.900,00$ \\
\hline Aluguel da Fábrica & $\mathrm{RS}$ & $4.500,00$ & $\mathrm{RS}$ & $54.000,00$ & $\mathrm{RS}$ & $54.000,00$ \\
\hline Salários a pagar & $\mathrm{RS}$ & $29.990,00$ & $\mathrm{RS}$ & $359.880,00$ & $\mathrm{RS}$ & $359.880,00$ \\
\hline Depreciação & $\mathrm{R} \$$ & $10.868,33$ & $\mathrm{RS}$ & $130.420,00$ & $\mathrm{RS}$ & $35.620,00$ \\
\hline Total dos Custos Fixos & RS & $62.725,43$ & RS & $752.705,20$ & RS & $657.905,20$ \\
\hline
\end{tabular}

Fonte: Autores (2020)

Vale-se ressaltar também que como os custos fixos dependem da depreciação, assim, o valor irá variar entre os dois cenários.

Quadro 8 - Custos variáveis

\begin{tabular}{|l|lr|}
\hline \multicolumn{3}{|c|}{ Custo Variável Unitário (por kg) } \\
\hline Aquisição de Matéria-Prima (Mulching) & R\$̦ & 0,17 \\
\hline Aquisição de Matéria-Prima (TNT) & R\$̦ & 0,15 \\
\hline Comissão do Corretor & & $2,5 \%$ \\
\hline
\end{tabular}

Fonte: Autores (2020) 
Quadro 9 - Despesas fixas mensais e anuais

\begin{tabular}{|c|c|c|c|c|}
\hline \multicolumn{5}{|c|}{ Despesas Fixas } \\
\hline Descrição & $\mathrm{Val}$ & or Mensal & & or Anual \\
\hline Contador (Terceirizado) & RȘ & 700,00 & $\mathrm{R} \$$ & $8.400,00$ \\
\hline Pró-labore da proprietária & RȘ & $3.000,00$ & $\mathrm{RS}$ & $36.000,00$ \\
\hline Contas a Pagar & $\mathrm{RS}$ & $2.226,00$ & RS & $26.712,00$ \\
\hline Total dos Despesas Fixas & $\mathrm{R} \$$ & $5.926,00$ & $\mathrm{R} \$$ & $71.112,00$ \\
\hline
\end{tabular}

Fonte: Autores (2020)

Quadro 10 - Despesas variáveis

\begin{tabular}{|l|cc|}
\hline \multicolumn{3}{|c|}{ Despesa Variável Unitária (por kg) } \\
\hline Frete de Carga Transportada (Mulching) & $\mathrm{R} \$$ & 0,20 \\
\hline Frete de Carga Transportada (TNT) & $\mathrm{R} \$$ & 0,40 \\
\hline
\end{tabular}

Fonte: Autores (2020)

A comercialização será realizada através do contato direto do cliente com a proprietária, que tem como pretensão futura realizá-la através de representantes. O telefone e o e-mail serão os principais veículos de contato entre os clientes e a empresa. A distribuição dos produtos será feita através de caminhões fretados, para todo o país, seguindo uma rota estratégica a fim de se reduzir o tempo gasto e quilometragem percorrida.

\subsection{Ponto de equilíbrio}

Seguindo-se com o estudo, foram efetuados os cálculos dos pontos de equilíbrio conforme as Equações 2, 4 e 5. Estes foram calculados para o cenário 1, tendo em vista ser o caso com o maior custo fixo e depreciação. Logo, se a empresa conseguir atingir o ponto de equilíbrio para esse cenário, entende-se que também alcançará resultados positivos no cenário 2 , dado que possui despendimentos financeiros menores.

Inicialmente, determinou-se a margem de contribuição unitária em reais para ambos os produtos, sendo esse resultado demonstrado no Quadro 11.

Quadro 11 - Margem de contribuição unitária dos produtos (R\$)

\begin{tabular}{|c|c|c|c|c|}
\hline \multicolumn{5}{|c|}{ Margem de contribuição unitária } \\
\hline Produto & $\begin{array}{l}\text { Preço de venda } \\
\text { unitário sem impostos }\end{array}$ & $\begin{array}{c}\text { Custo variável } \\
\text { unitário }\end{array}$ & $\begin{array}{c}\text { Despesa variável } \\
\text { unitária }\end{array}$ & $\begin{array}{c}\text { Margem de contribuição } \\
\text { unitária }\end{array}$ \\
\hline TNT & 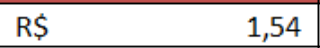 & $\mathrm{R} \$ \quad \quad 0,15$ & 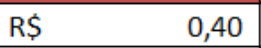 & $\mathrm{R} \$+0,98$ \\
\hline Mulching & $\mathrm{RS} \quad 1,63$ & 0,17 & 0,20 & 1,25 \\
\hline \multicolumn{4}{|c|}{ Margem de contribuição unitária total } & 2,2352 \\
\hline
\end{tabular}

Fonte: Autores (2020)

Além disso, foi estipulado o valor da margem de contribuição unitária em porcentagem, através da Equação 3. 


$$
\text { MCT } \%=\frac{2,2353}{1,7+1,8}=63,8629 \%
$$

Com esses valores já conhecidos, a etapa seguinte consistiu na determinação do PEC anual da organização, conforme a Equação 2.

$$
P E C=\frac{752.705,20+71.112,00}{63,8629 \%}=R \$ 1.289 .978,61
$$

O resultado de $\mathrm{R} \$ 1.289 .978,61$ significa que este é o valor mínimo de faturamento anual que garante que a empresa cubra seus gastos, não obtendo prejuízo.

Através da Equação 4, foi calculado o PEE, onde foi escolhido um lucro mínimo anual desejado de $\mathrm{R} \$ 200.000,00$.

$$
P E E=\frac{752.705,20+71.112,00+200.000,00}{63,8629 \%}=R \$ 1.603 .149,70
$$

O PEE de R \$ 1.603.149,70 é o valor mínimo de faturamento que garante que a empresa cubra seus gastos e ainda obtenha o lucro mínimo que deseja.

Utilizando-se a Equação 5, foi possível obter o PEF anual da empresa, considerando a depreciação para o cenário 1 .

$$
P E F=\frac{752.705,20+71.112,00-130.420,00}{63,8629 \%}=R \$ 1.085 .759,75
$$

O resultado demonstra um saldo positivo de $\mathrm{R} \$ 1.085 .759,75$, sendo esse valor o faturamento anual da empresa necessário para pagar seus gastos fixos, não sendo considerado a depreciação.

\subsection{DRE}

Para fornecer informações úteis que auxiliem na tomada de decisões, elaborou-se o DRE através dos demais parâmetros estipulados anteriormente, sendo esse a taxa do imposto Simples Nacional, a receita e os gastos fixos e variáveis. O resultado obtido para o DRE de cada cenário é apresentado na Tabela 1 e 2, respectivamente. 
Tabela 1 - DRE cenário 1

\begin{tabular}{|c|c|c|c|}
\hline \multicolumn{4}{|c|}{ DRE - CENÁRIO 1} \\
\hline & Receita/Faturamento Bruto (RB) & $\mathrm{RS}$ & $1.785 .600,00$ \\
\hline$(-)$ & Deduções/Impostos sobre Vendas (DIV) & $\mathrm{RS}$ & $199.987,20$ \\
\hline$\Leftrightarrow$ & Receita/Faturamento Liquido (RL) & $\mathrm{RS}$ & $1.585 .612,80$ \\
\hline$(-)$ & Custos dos Produtos Vendidos (CPV) & $\mathrm{R} \$$ & $277.593,96$ \\
\hline & Custo Variável Total (CVT) & $\mathrm{R} \$$ & $18.393,96$ \\
\hline & Despesa Variável Total (DVT) & $\mathrm{R} \$$ & $259.200,00$ \\
\hline$\Leftrightarrow$ & Margem de Contribuição Total (MCT) & $\mathrm{R} \$$ & $1.308 .018,84$ \\
\hline$(-)$ & Gastos Fixos do Período (GFP) & $\mathrm{RS}$ & $823.817,20$ \\
\hline & Custo Fixo Total (CFT) & $\mathrm{RS}$ & $752.705,20$ \\
\hline & Despesa Fixa Total (DFT) & $\mathrm{R} \$$ & $71.112,00$ \\
\hline$\Leftrightarrow$ & Lucro Líquido Antes do Imposto de Renda (LAIR) & $\mathrm{R} \$$ & $484.201,64$ \\
\hline$(-)$ & Imposto de Renda (IR) & $\mathrm{RS}$ & - \\
\hline$\Leftrightarrow$ & Lucro Líquido (LL) & R\$ & $484.201,64$ \\
\hline
\end{tabular}

Fonte: Autores (2020)

Tabela 2 - DRE cenário 2

\begin{tabular}{|c|c|c|c|}
\hline \multicolumn{4}{|c|}{ DRE - CENÁRIO 2} \\
\hline & Receita/Faturamento Bruto (RB) & $R \$$ & $1.785 .600,00$ \\
\hline$(-)$ & Deduções/Impostos sobre Vendas (DIV) & $\mathrm{R} \$$ & $199.987,20$ \\
\hline$\Leftrightarrow$ & Receita/Faturamento Líquido (RL) & $\mathrm{R} \$$ & $1.585 .612,80$ \\
\hline$(-)$ & Custos dos Produtos Vendidos (CPV) & $\mathrm{R} \$$ & $277.593,96$ \\
\hline & Custo Variável Total (CVT) & $\mathrm{R} \$$ & $18.393,96$ \\
\hline & Despesa Variável Total (DVT) & $R \$$ & $259.200,00$ \\
\hline$(=)$ & Margem de Contribuição Total (MCT) & RS & $1.308 .018,84$ \\
\hline$(-)$ & Gastos Fixos do Período (GFP) & $\mathrm{RS}$ & $729.017,20$ \\
\hline & Custo Fixo Total (CFT) & $\mathrm{R} \$$ & $657.905,20$ \\
\hline & Despesa Fixa Total (DFT) & $\mathrm{R} \$$ & $71.112,00$ \\
\hline$\Leftrightarrow$ & Lucro Líquido Antes do Imposto de Renda (LAIR) & $\mathrm{R} \$$ & $579.001,64$ \\
\hline$(-)$ & Imposto de Renda (IR) & $R \$$ & - \\
\hline$(=)$ & Lucro Líquido (LL) & $\mathrm{R} \$$ & $579.001,64$ \\
\hline
\end{tabular}

Fonte: Autores (2020)

Observa-se que o LAIR consiste no mesmo valor do LL, visto que o Imposto de Renda já é incorporado na alíquota do Simples Nacional deduzida no início da DRE.

\subsection{Fluxo de Caixa Livre}

Para a elaboração do FLC, utilizou-se um período de 10 anos, visto o tempo máximo de depreciação dos ativos da organização. Logo, foi desenvolvido o FCL englobando nos cinco primeiros anos os dados referentes a esse período e nos últimos cinco anos as demais informações necessárias, onde é visto a diferença de lucro líquido entre os cenários decorrentes dos valores de custo fixo e depreciação para cada caso. O Valor Residual dos Equipamentos é apresentado no Quadro 13, enquanto os Quadros 14 e 15 apresentam o resultado alcançado. Observa-se que o LAJIR se igualou ao LADIR, devido ao Imposto de Renda já ter sido considerado no início dos cálculos, dado que ele está incluído no valor do Simples Nacional. 
Quadro 12 - Cálculo do Valor Residual dos Equipamentos

\begin{tabular}{|l|rr|rr|}
\hline \multicolumn{1}{|c|}{ Equipamento } & \multicolumn{2}{c|}{ Valor de Aquisição } & \multicolumn{2}{c|}{ Valor Residual } \\
\hline Máquina de corte & $\mathrm{R} \$$ & $4.200,00$ & $\mathrm{R} \$$ & $1.500,00$ \\
\hline Balança Industrial & $\mathrm{R} \$$ & $1.700,00$ & $\mathrm{R} \$$ & 800,00 \\
\hline Esteira Industrial para transporte & $\mathrm{R} \$$ & $7.800,00$ & $\mathrm{R} \$$ & $3.000,00$ \\
\hline Kit (Moinho + Ventoinhas + Centrifugador) & $\mathrm{R} \$$ & $250.000,00$ & $\mathrm{R} \$$ & $80.000,00$ \\
\hline Amolador de Lâminas para Moinho & $\mathrm{R} \$$ & $2.500,00$ & $\mathrm{R} \$$ & $1.000,00$ \\
\hline Prensa Enfardadeira & $\mathrm{R} \$$ & $90.000,00$ & $\mathrm{R} \$$ & $40.000,00$ \\
\hline Caminhão & $\mathrm{R} \$$ & $474.000,00$ & $\mathrm{R} \$$ & $200.000,00$ \\
\hline Materiais para escritório & $\mathrm{R} \$$ & $4.059,00$ & $\mathrm{R} \$$ & $2.000,00$ \\
\hline \multicolumn{2}{|c|}{ Total } & & & $\mathrm{R} \$ 328.300,00$ \\
\hline
\end{tabular}

Fonte: Autores (2020) 
Quadro 13 - FCL

\begin{tabular}{|c|c|c|c|c|c|c|c|c|}
\hline & \multicolumn{2}{|r|}{ ANOO } & \multicolumn{2}{|r|}{ ANO 1} & \multicolumn{2}{|r|}{ ANO 2} & \multicolumn{2}{|r|}{ ANO 3} \\
\hline (+) Receita Bruta & $\mathrm{R}$ Ș & - & $R S$ & $1.785 .600,00$ & $\mathrm{RST}$ & $1.785 .600,00$ & RS : & $1.785 .600,00$ \\
\hline (-) Impostos e Deduções sobre Receita Bruta & RȘ & - & $\mathrm{RST}$ & $199.987,20$ & $\mathrm{RSS}$ & $199.987,20$ & $\mathrm{RSS}$ & $199.987,20$ \\
\hline (=) Receita Ĺquida & $\mathrm{RS}$ & - & $\mathrm{RS}$ & $1.585 .612,80$ & $\mathrm{RS}$ & $1.585 .612,80$ & $\mathrm{RS}$ & $1.585 .612,80$ \\
\hline (-) Custos Variáveis Desembolsáveis & $\mathrm{RS}$ & - & $\mathrm{RS}$ & $18.393,96$ & $\mathrm{RS}$ & $18.393,96$ & $\mathrm{RSS}$ & $18.393,96$ \\
\hline (-) Despesas Variáveis Desembolsáveis & $\mathrm{RS}$ & - & $\mathrm{RSS}$ & $259.200,00$ & $\mathrm{RS}$ & $259.200,00$ & $\mathrm{RS}$ & $259.200,00$ \\
\hline (=) Margem de Contribuição Total & $\mathrm{RS}$ & - & $\mathrm{RS}$ & $1.308 .018,84$ & $\mathrm{RS}$ & $1.308 .018,84$ & $\mathrm{RS}$ & $1.308 .018,84$ \\
\hline (-) Custos Fixos Desembolsáveis (sem Depreciação) & $\mathrm{RST}$ & - & RȘ & $752.705,20$ & $\mathrm{RSS}$ & $752.705,20$ & RȘ & $752.705,20$ \\
\hline (-) Despesas Fixas Desembolsáveis (sem Depreciação) & RȘ & - & RȘ & $71.112,00$ & $\mathrm{RST}$ & $71.112,00$ & $\mathrm{RST}$ & $71.112,00$ \\
\hline (=) Lucro Operacional Antes de Juros, Imposto de Renda, Depreciação e Amortização (LAIIDA ou EBITDA) & $\mathrm{R}$ Ș & - & RȘ & $484.201,64$ & $\mathrm{RST}$ & $484.201,64$ & $\mathrm{RST}$ & $484.201,64$ \\
\hline (-) Depreciação (no simples nacional não tem o beneficio fiscal da depreciação) & RȘ & - & RȘ & $130.420,00$ & $\mathrm{RSS}$ & $130.420,00$ & $\mathrm{RS}$ & $130.420,00$ \\
\hline (=) Lucro Operacional Antes de Juros e Imposto de Renda (LAIR ou EBT) & $\mathrm{RS}$ & - & $\mathrm{RS}$ & $353.781,64$ & $\mathrm{RSS}$ & $353.781,64$ & $\mathrm{RS}$ & $353.781,64$ \\
\hline (-) Impostos Incidentes sobre Renda (ex: IR e CSLL) (no simples nacional o IR =0) & RS & - & $\mathrm{RST}$ & & $\mathrm{RSS}$ & - & RȘ & \\
\hline (=) Lucro Operacional Líquido Depois do Imposto de Renda (LADIR ou NOPLAT) & $\mathrm{R}$ Ș & - & $\mathrm{RST}$ & $353.781,64$ & $\mathrm{RST}$ & $353.781,64$ & $\mathrm{RSS}$ & $353.781,64$ \\
\hline (+) Depreciação & $\mathrm{RS}$ & - & $\mathrm{RSS}$ & $130.420,00$ & $\mathrm{RSS}$ & $130.420,00$ & $\mathrm{RS}$ & $130.420,00$ \\
\hline (=) Fluxo de Caixa Operacional & $\mathrm{RST}$ & - & RS & $484.201,64$ & RS & $484.201,64$ & $\mathrm{R} \$$ & $484.201,64$ \\
\hline (-) Investimento em Ativos Permanentes & $\mathrm{RSS}$ & $944.375,00$ & $\mathrm{RST}$ & & $\mathrm{RSS}$ & - & RSS & - \\
\hline (-) Necessidade de Capital de Giro & RS & - & $\mathrm{RS}$ & $48.416,00$ & $\mathrm{RSS}$ & - & $\mathrm{RSS}$ & - \\
\hline (+) Capital de Giro Recuperado & $\mathrm{R}$ \$̦ & - & $\mathrm{RSS}$ & - & $\mathrm{RSS}$ & - & $\mathrm{RST}$ & - \\
\hline (+) Valor Residual do Ativo Vendido & $\mathrm{RS}$ & - & $\mathrm{RSS}$ & - & $\mathrm{RS}$ & - & $\mathrm{RSS}$ & - \\
\hline (=) Fluxo de Caixa Livre & -RS & $944.375,00$ & $\mathrm{RS}$ & $435.785,64$ & $\mathrm{RS}$ & $484.201,64$ & $\mathrm{R} \$$ & $484.201,64$ \\
\hline
\end{tabular}

Fonte: Autores (2020) 
Quadro 14 - Continuação FCL

\begin{tabular}{|c|c|c|c|c|c|c|c|c|c|c|c|c|c|}
\hline \multicolumn{2}{|r|}{ ANO 4} & \multicolumn{2}{|r|}{ ANO 5} & \multicolumn{2}{|r|}{ ANO 6} & \multicolumn{2}{|r|}{ ANO 7} & \multicolumn{2}{|r|}{ ANO 8} & \multicolumn{2}{|r|}{ ANO 9} & \multicolumn{2}{|r|}{ ANO 10} \\
\hline RȘ & $1.785 .600,00$ & $\mathrm{RSS}$ & $1.785 .600,00$ & $\mathrm{RS}$ & $1.785 .600,00$ & $\mathrm{RS}$ & $1.785 .600,00$ & $\mathrm{RS}$ & $1.785 .600,00$ & $\mathrm{RS}$ & $1.785 .600,00$ & $\mathrm{RS}$ & $1.785 .600,00$ \\
\hline $\mathrm{RS}$ & $199.987,20$ & $\mathrm{RSS}$ & $199.987,20$ & $\mathrm{RSS}$ & $199.987,20$ & $\mathrm{R} \$$ & $199.987,20$ & $\mathrm{RS}$ & $199.987,20$ & $\mathrm{RS}$ & $199.987,20$ & $\mathrm{RS}$ & $199.987,20$ \\
\hline RȘ & $1.585 .612,80$ & $R S$ & $1.585 .612,80$ & $\mathrm{RS}$ & $1.585 .612,80$ & $\mathrm{RS}$ & $1.585 .612,80$ & $\mathrm{RS}$ & $1.585 .612,80$ & $\mathrm{RS}$ & $1.585 .612,80$ & $\mathrm{RS}$ & $1.585 .612,80$ \\
\hline $\mathrm{R} \$$ & $18.393,96$ & $R S$ & $18.393,96$ & $\mathrm{RSS}$ & $18.393,96$ & $\mathrm{R} \$$ & $18.393,96$ & $\mathrm{RSS}$ & $18.393,96$ & $\mathrm{RSS}$ & $18.393,96$ & $\mathrm{RS}$ & $18.393,96$ \\
\hline $\mathrm{RS}$ & $259.200,00$ & RSS & $259.200,00$ & $\mathrm{RSS}$ & $259.200,00$ & $\mathrm{RSS}$ & $259.200,00$ & $\mathrm{RS}$ & $259.200,00$ & $\mathrm{RS}$ & $259.200,00$ & $\mathrm{RS}$ & $259.200,00$ \\
\hline $\mathrm{RS}$ & $1.308 .018,84$ & $\mathrm{RS}$ & $1.308 .018,84$ & $\mathrm{R} \$$ & $1.308 .018,84$ & $\mathrm{RS}$ & $1.308 .018,84$ & $\mathrm{RSS}$ & $1.308 .018,84$ & $\mathrm{R} \$$ & $1.308 .018,84$ & $\mathrm{RS}$ & $1.308 .018,84$ \\
\hline $\mathrm{RS}$ & $752.705,20$ & $\mathrm{RS}$ & $752.705,20$ & $\mathrm{RSS}$ & $657.905,20$ & $\mathrm{R} \$$ & $657.905,20$ & $\mathrm{RS}$ & $657.905,20$ & $\mathrm{RS}$ & $657.905,20$ & $\mathrm{RS}$ & $657.905,20$ \\
\hline $\mathrm{RS}$ & $71.112,00$ & $\mathrm{RS}$ & $71.112,00$ & $\mathrm{RS}$ & $71.112,00$ & $\mathrm{R} \$$ & $71.112,00$ & $\mathrm{RSS}$ & $71.112,00$ & $\mathrm{RSS}$ & $71.112,00$ & $\mathrm{RS}$ & $71.112,00$ \\
\hline $\mathrm{RS}$ & $484.201,64$ & $\mathrm{RS}$ & $484.201,64$ & $\mathrm{RSS}$ & $579.001,64$ & $\mathrm{R} \$$ & $579.001,64$ & $\mathrm{RS}$ & $579.001,64$ & $\mathrm{RS}$ & $579.001,64$ & $\mathrm{RS}$ & $579.001,64$ \\
\hline $\mathrm{RS}$ & $130.420,00$ & $\mathrm{RS}$ & $130.420,00$ & $\mathrm{RS}$ & $35.620,00$ & $\mathrm{R} \$$ & $35.620,00$ & $\mathrm{RS}$ & $35.620,00$ & $\mathrm{RSS}$ & $35.620,00$ & $\mathrm{R} \$$ & $35.620,00$ \\
\hline $\mathrm{RS}$ & $353.781,64$ & $\mathrm{RS}$ & $353.781,64$ & $\mathrm{RSS}$ & $543.381,64$ & $\mathrm{R} \$$ & $543.381,64$ & $\mathrm{RS}$ & $543.381,64$ & $\mathrm{RS}$ & $543.381,64$ & $\mathrm{RS}$ & $543.381,64$ \\
\hline $\mathrm{RS}$ & - & $\mathrm{RS}$ & - & $\mathrm{RS}$ & - & $\mathrm{RS}$ & - & $\mathrm{RS}$ & - & $\mathrm{RS}$ & - & $\mathrm{RS}$ & - \\
\hline $\mathrm{RS}$ & $353.781,64$ & $\mathrm{RS}$ & $353.781,64$ & $\mathrm{RSS}$ & $543.381,64$ & $\mathrm{R} S$ & $543.381,64$ & $\mathrm{RS}$ & $543.381,64$ & $\mathrm{RS}$ & $543.381,64$ & $\mathrm{RS}$ & $543.381,64$ \\
\hline $\mathrm{RS}$ & $130.420,00$ & $\mathrm{RSS}$ & $130.420,00$ & $\mathrm{RSS}$ & $35.620,00$ & $\mathrm{R} \$$ & $35.620,00$ & $\mathrm{RSS}$ & $35.620,00$ & $\mathrm{RSS}$ & $35.620,00$ & $\mathrm{R} S$ & $35.620,00$ \\
\hline $\mathrm{R} \$$ & $484.201,64$ & $\mathrm{RS}$ & $484.201,64$ & $\mathrm{RS}$ & $579.001,64$ & $\mathrm{R} S$ & $579.001,64$ & $\mathrm{RS}$ & $579.001,64$ & $\mathrm{RS}$ & $579.001,64$ & $\mathrm{RS}$ & $579.001,64$ \\
\hline $\mathrm{RS}$ & - & $\mathrm{RS}$ & - & $\mathrm{RS}$ & - & $\mathrm{RS}$ & - & $\mathrm{RSS}$ & - & $\mathrm{RSS}$ & - & $\mathrm{R} \$$ & - \\
\hline $\mathrm{RS}$ & - & $\mathrm{RS}$ & - & $\mathrm{RS}$ & - & $\mathrm{RS}$ & - & $\mathrm{RSS}$ & - & $\mathrm{RS}$ & - & $\mathrm{RS}$ & - \\
\hline $\mathrm{RS}$ & - & $\mathrm{RS}$ & - & $\mathrm{R} S$ & - & $\mathrm{R} \$$ & - & $\mathrm{RSS}$ & - & $\mathrm{RSS}$ & - & $\mathrm{RSS}$ & $48.416,00$ \\
\hline $\mathrm{RS}$ & - & $\mathrm{RS}$ & - & $\mathrm{RS}$ & - & $\mathrm{R} \$$ & - & $\mathrm{RSS}$ & - & $\mathrm{RSS}$ & - & & 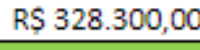 \\
\hline $\mathrm{RS}$ & $484.201,64$ & $\mathrm{RS}$ & $484.201,64$ & $\mathrm{RS}$ & $579.001,64$ & $\mathrm{R} \$$ & $579.001,64$ & $\mathrm{R} \$$ & $579.001,64$ & $\mathrm{RS}$ & $579.001,64$ & $\mathrm{RS}$ & $955.717,64$ \\
\hline
\end{tabular}

Fonte: Autores (2020) 


\subsection{Lucratividade e rentabilidade}

Para a análise do nível de sucesso do investimento, calculou-se a lucratividade e rentabilidade anuais para cada cenário, de acordo com as Equações 7 e 8.

$$
\begin{aligned}
& \text { Lucratividade CENÁRIO } 1=\frac{484.201,64}{1.785 .600,00}=27,12 \% \\
& \text { Lucratividade CENÁRIO } 2=\frac{579.001,64}{1.785 .600,00}=32,42 \% \\
& \text { Rentabilidade CENÁRIO } 1=\frac{484.201,64}{944.375,00}=51,27 \% \\
& \text { Rentabilidade CENÁRIO } 2=\frac{579.001,64}{944.375,00}=61,31 \%
\end{aligned}
$$

Logo, a lucratividade média de ambos os cenários indicou $29,77 \%$ de lucro sobre as vendas e a rentabilidade média apresentou $56,29 \%$ de rendimento do capital investido.

\subsection{VPL, TIR e pay-back descontado}

Com os resultados obtidos no FCL, elaborou-se os cálculos dos indicadores de viabilidade econômica VPL e TIR, apresentados no Quadro 16.

\begin{tabular}{|l|r|}
\hline \multicolumn{2}{|c|}{ Quadro 15 - Cálculo do VPL e TIR } \\
\hline COC $=$ & $3 \%$ \\
\hline VPL= & $\mathrm{R} \$ 3.793 .776,45$ \\
\hline TIR= & $50,17 \%$ \\
\hline
\end{tabular}

Fonte: Autores (2020)

O resultado obtido para o pay-back descontado é apresentado no Quadro 17.

Quadro 16 - Cálculo do pay-back descontado

\begin{tabular}{|l|rr|}
\hline Para $n=1$ & -R\$ & $521.282,15$ \\
\hline Para $n=2$ & -R\$ & $64.875,66$ \\
\hline Para $n=3$ & R\$ & $378.237,43$ \\
\hline
\end{tabular}

Fonte: Autores (2020) 
O Quadro 17 mostra que o VPL se torna positivo em $n=3$, o que significa que o prazo de retorno de investimento (pay-back descontado) é de 3 anos. Visto que $\mathrm{K}=3 \%$, como a TIR 49\% > K e o VPL 3.419.269,46 > 0, então o projeto é economicamente viável.

\subsection{Análise de riscos do projeto}

Com relação aos riscos do projeto, podemos destacar:

- A variação no custo da mão de obra e dos insumos, que podem acarretar um aumento do preço de venda do produto e, por consequência, a insatisfação dos clientes;

- Fácil fabricação dos produtos, pois não exige mão de obra qualificada, nem alta tecnologia, o que pode tornar-se alvo de possíveis concorrentes;

- Dependência direta de um setor muito instável, que é a agricultura. Em períodos de baixa, a produção na agricultura será menor, consequentemente a quantidade de resíduos gerados, que consiste na matéria prima da fábrica, também.

\section{Conclusão}

Conforme o estudo apresentado, pôde-se observar que o objetivo proposto foi atingido, visto que foi realizada a análise de viabilidade econômica para o processo de reaproveitamento de materiais, TNT e Mulching, provindos de empresas agrícolas.

Para isto, ocorreu o estudo de conceitos e métodos associados a análise financeira de investimentos. Dentre estes os conceitos básicos de investimento, receita, custo e despesa. Posteriormente retratou-se as definições de Ponto de Equilíbrio, incluindo o Contábil, o Econômico e o Financeiro de modo que fosse possível identificar a saúde financeira da empresa.

Adiante, ocorreu o estudo do DRE e do FLC para possibilitar a análise da situação financeira da organização, bem como dos índices de lucratividade e rentabilidade para indicar o nível de sucesso do investimento. Por fim pesquisou-se sobre o VPL, TIR e payback descontado para avaliar a viabilidade econômica.

Frente aos resultados alcançados, visualizou-se PEC de $\mathrm{R} \$ 1.289 .978,61$ como faturamento mínimo para se cobrir os gastos, PEE de R\$1.603.149,70 que garante ainda que se obtenha o lucro mínimo desejado, PEF de $\mathrm{R} \$$ 1.085.759,75 (ambos calculados para 
o cenário 1), e como principais métricas apresentadas obteve-se lucratividade média de $29,77 \%$, rentabilidade de 56,29\%, TIR 50,17\% > 3\%, VPL R $\$ 3.793 .776,45>0$ e payback descontado de 3 anos. Com isso, foi possível visualizar e denotar a viabilidade financeira do investimento requerido.

Diante do elevado consumismo intrínseco a atual sociedade, o presente trabalho possui relevância não apenas na propagação e aquisição do conhecimento no que diz respeito a análise financeira de investimentos, como também retrata a importância da elaboração de processos industriais capazes de proporcionar a reutilização de materiais suscetíveis a reciclagem.

Como trabalho futuro propõe-se a análise da viabilidade do investimento utilizando-se métodos complementares ao VPL, como exemplo a análise probabilística realizada por meio da simulação do Método de Monte Carlos (MMC). Deste modo, com a utilização do MMC será possível quantificar e visualizar as possibilidades de ocorrências de cenários futuros, auxiliando no processo gerencial de tomada de decisão.

\section{REFERÊNCIAS}

ASSAF NETO, Alexandre; LIMA, Fabiano Guasti. Curso de administração financeira. 3.ed. São Paulo: Atlas, 2014.

CINQUETTI, Heloisa Sisla. Lixo, resíduos sólidos e reciclagem: uma análise comparativa de recursos didáticos. Educar. Curitiba, v. n. 23 p. 307-333, Editora UFPR, 2004.

EMBRAPA. Uso de plástico na agricultura protegida. 01 de nov. de 2015. Disponível em: <https://www.embrapa.br/busca-de-noticias/-/noticia/3230175/uso-do-plastico-na-agricultura-protegida>. Acesso em: 27 de mai. de 2020.

GERHARDT, Tatiana Engel; SILVEIRA, Denise Tolfo. Métodos de pesquisa. Porto Alegre: Editora da UFRGS, 2009.

GIL, Atonio Carlos. Métodos e técnicas de pesquisa social. 6 ed. São Paulo: Atlas, 2008.

GOTO, R. Plasticultura nos trópicos: uma avaliação técnico-econômica. Horticultura Brasileira, Brasília, v. 15, p. 163-165, 1997.

IPEA. Apenas 13\% dos resíduos sólidos urbanos no país vão para reciclagem. 25 de jan. de 2017. Disponível em:<https://www.ipea.gov.br/portal/index.php?option=com_content\&view=article\&id=29296:apenas-13- 
dos-residuos-urbanos-no-pais-vao-para-reciclagem\&catid=1:dirur\&directory=1>. Acesso em: 27 de mai. de 2020.

MARION, José Carlos; RIBEIRO, Osni Moura. Introdução à contabilidade gerencial. São Paulo: Saraiva, 2011.

MARTINS, Eliseu. Contabilidade de custos. 9 ed. São Paulo: Atlas, 2003.

OLIVEIRA, Estevani Pereira; APOLINÁRIO, Valdênia; SILVA, Maria Lussieu. Sistema Produtivo e Inovativo Local: O APL da Fruticultura de Melão de Mossoró/Baraúna, no Rio Grande do Norte Brasil. Conferência Internacional LALICS 2013. Rio de Janeiro, 2013.

PADOVEZE, Clóvis Luís. Curso básico gerencial de custos. 2 ed. São Paulo: Cengage Learning, 2006.

PRODANOV, Cleber Cristiano; FREITAS, Ernani Cesar de. Metodologia do trabalho científico [recurso eletrônico]: métodos e técnicas da pesquisa e do trabalho acadêmico. 2 ed. Novo Hamburgo: Feevale, 2013.

SAMANEZ, Carlos Patrício. Matemática Financeira: Aplicações à Análise de Investimentos. São Paulo: Prentice Hall, 2002.

SANTOS, Emanuel Eduardo Paiva. Análise custo-benefício da utilização de filmes biodegradáveis vs filmes de polietileno: uma aplicação concreta a uma cultura de ciclo curto. Dissertação para a obtenção do Grau de Mestre em Engenharia Agronómica. Universidade de Lisboa. Lisboa, 2014.

SILVA, Romero Rossano Tertulino. A importância de Mossoró para o contexto econômico potiguar. Revista Eletrônica de Petróleo e Gás. Ano 3, n. 2, p. 53-63. Mossoró, 2015.

YIN, Robert K. Estudo de caso: planejamento e métodos. 2 ed. Porto Alegre: Bookman, 2001. 